nucleate. Sluder et al. ${ }^{8}$ find that the centrosomes in enucleated sea urchin embryos undergo division in a precise $1 \rightarrow 2 \rightarrow 4 \rightarrow 8$ fashion, with a slightly slower cycle time than control embryos. Electron microscopy reveals that these centrosomes contain the normal complement of centrioles, suggesting that the centrosomes undergo normal division rather than fragmentation. Although the enucleated sea urchin embryos do not undergo cytokinesis, cleavage furrows are initiated to a variable degree. By contrast, starfish embryos treated with aphidicolin can undergo many rounds of cytokinesis.

It is worth considering the role of the centrosome as a cytoplasmic organizer in a developmental context. The nuclei that migrate to the posterior pole of the
Drosophila embryo must interact with specifically localized determinants that ultimately cause them to become pole cells. The finding that pole cells can form in the absence of nuclei suggests that some of these determinants become incorporated specifically into the pole-cell lineage by virtue of an interaction with the centrosome or its associated microtubule networks. In any case, it is clear that an understanding of the spatial and temporal organization of the early Drosophila embryo cytoplasm is integral to our understanding of the process of pattern formation.

Douglas R. Kellogg is in the Department of Biochemistry and Biophysics, University of California School of Medicine, San Francisco, California 94143-0448, USA.

\title{
Less thick than a brick
}

\section{Paul Calvert}

INTELLIGENT materials seem to have become a new hot topic: the US army is about to inaugurate a research initiative to develop them; and recent meetings in Japan and the United States indicate dawning interest amongst materials scientists. But although several specific examples were presented at the latest meeting*, it is still not obvious exactly what constitutes an intelligent material. Nevertheless, the concept is attractive and clearly leads in the right direction for the achievement of a higher level of integration between machines and the materials used to construct them.

The hydrogels are one group of materials that have some of the right characteristics. These are water-swollen crosslinked polymers, usually modified acrylates or acrylamides. The physics of these systems has been studied in detail by Tanaka and co-workers ${ }^{1}$ and other groups are working on devices ${ }^{2}$. If the gel contains anionic groups such as carboxylic acids, the equilibrium swelling is very sensitive to the ionic strength of the surrounding liquid. By moving the gel from a salt solution to water, and by applying an electric field to drive out the counter ion, the gel can be made to contract. The action is superficially similar to that of muscle. This ability of a material to respond to a change in its surroundings must be part of the concept of intelligence. In practical terms, the response is slow (taking 1-10 s, much more than the $10-100 \mathrm{~ms}$ taken by muscle fibres) and the muscle action is rather weak $\left(1 \mathrm{~mW} \mathrm{~cm}^{-3}\right.$; muscle delivers $1 \mathrm{~W}$ $\mathrm{cm}^{-3}$ ). But work is continuing on devices for robotics, for controlled drug delivery and for variable porosity membranes.

Craig Rogers (Virginia Polytechnic) has

* International Workshop on Intelligent Materials, Tsukuba. 15-17 March 1989. been working on 'smart systems' which include the ability to damp vibrations actively. A framework of struts with strain gauges and motors that can change the length of a strut, can be programmed to counteract vibrations of a particular frequency. It tackles a problem similar to that of walking without spilling a cup of coffee. This work has now been extended to studies of composite materials containing fibres of shape-memory alloy. Shapememory alloys undergo large, reversible changes of shape when heated through a transition temperature. By selective electrical heating of some of the fibres, the stiffness and vibrational modes of the beam can be tuned ${ }^{3}$.

Danilo de Rossi (Università degli Studi di Pisa) has been working on an artificial skin which could be used for robots. Such a material should be able to sense changes in temperature and heat flow, such as allow us to distinguish thermal conductivity when we touch cold metal or plastic. It should also be able to sense the full six components of stress, not just the pressure perpendicular to the skin, and have good spatial resolution. In this way one would hope to be able to distinguish surface characteristics such as roughness, friction and stickiness. In skin the response may be associated with the piezoelectric behaviour of keratin plus a pressure sensitivity in the underlying dermis.

Rossi has been investigating various types of sensors, including a pseudoepidermis of multiple layers of piezoelectric polymers such as polyvinylidene fluoride. The dermis is modelled by a layer of fibre-reinforced polyelectrolyte gel with embedded electrodes acting as sensors for pressure and strain rate. This material shows a further aspect that one might associate with intelligence, the ability to sense changes in the surroundings using multiple channels of information ${ }^{4}$.

A hollow composite beam can be filled with electro-rheological fluid which becomes rigid under an applied field and so make the beam stiffer (M.V. Ghandi, Michigan State University). Coupled with sensors, this would allow the beam to change properties in response to changes in load. Piezoelectric materials acquire a voltage when strained, and contract, expand or bend when charged electrically. They are thus an obvious starting point for assembling an intelligent structure. R. E. Newnham (Pennsylvania State University) is working on combinations of piezoelectric sensors and actuators which will contract away from a surface when they touch it, so giving the hard material an apparently soft touch.

High-performance composite materials for aircraft are mainly carbon-fibrereinforced polymer. By incorporating a few silica fibres one can use spectroscopic techniques to probe the degree of cure, degradation or stress levels inside the material. In principle this information could be used at least to make the material declare its imminent failure, if not to initiate its own repair.

From these studies one can begin to see what should be the properties of an intelligent material. It should be able to sense changes in its environment in some detail. It should contain some actuator function to allow a response to those changes. The link should be governed by a logic system although the 'brain' is not necessarily embedded in the material. Obvious models can be found in biological materials and several were discussed at the meeting. One characteristic is the ability to restructure in response to external stress, as bone remodels when continually loaded non-uniformly. Also, intelligent materials should be able to remodel in the face of high local stresses, corrosion or developing fatigue. This means that we should start to think of materials not necessarily as closed systems, but as being able to change composition or to grow.

Whether 'intelligent' or 'smart' are the right descriptions is an open question, as is the appropriateness of the term 'materials'. The last word should probably go to Humpty Dumpty": "When I use a word, it means just what I choose it to mean neither more nor less"

Paul Calvert is in the Arizona Materials Labora. tory, University of Arizona, Tucson, Arizona 85712, USA.

\footnotetext{
1. Tanaka, T. et al. Phys. Rev. Lett. 45, 1636-1639 (1980). 2. Osada, Y., Kishi, R. \& Hasebe, M. J. Polymer Sci. Lett. 25 481-485 (1987).

3. Rogers, C.A. (ed.) Smart Materials, Structures and Mathematical Issues (Technomic, 1989)

4. DeRossi, D. Domenici, C. \& Chiarelli, P in Sensors and Sensory Systems for Advanced Robots (ed. Dario, P.) (NATO ASI Series, Springer, Berlin, 1988)

5. Carroll, L. Through the Looking Glass (Macmillan, London,
} 1.956) 